15

\title{
Исследование коэффициента диффузии в тонких изделиях из пористых материалов
}

\author{
(C) В.П. Беляев, С.В. Мищенко, П.С. Беляев \\ Тамбовский государственный технический университет, \\ 392000 Тамбов, Россия \\ e-mail: bps800@yandex.ru
}

Поступило в Редакцию 4 декабря 2018 г.

В окончательной редакции 25 марта 2019 г.

Принято к публикации 15 апреля 2019 г.

Рассмотрен новый метод исследования процессов массопереноса в пористых средах, позволяющий проводить оперативный контроль коэффициента диффузии в тонких изделиях без их разрушения и в отсутствие предварительной градуировки применяемого измерителя концентрации диффузанта. Метод обеспечил повышение точности измерения искомой характеристики массопереноса за счет возможности выбора входящих в расчетное выражение измеряемых параметров на участках статической характеристики преобразователя с высокой чувствительностью и помехозащищенностью.

Ключевые слова: диффузия, пористый материал, неразрушающий контроль, оперативность.

DOI: $10.21883 /$ JTF.2019.10.48184.420-18

\section{Введение}

Большинство экспериментальных данных по коэффициентам диффузии в настоящее время определяется на установках значительной сложности и низкой производительности, не позволяющих проводить оперативную оценку качества готовых изделий. Для проведения исследований необходимо использование образцов определенной формы и размеров, получаемых путем разрушения готовых изделий или их изготовления по рецептуре и технологиям, соответствующим реальному производству. В процессе исследований часто необходимо осуществлять разрушение самого исследуемого образца для измерения пространственных распределений диффундирующих веществ (диффузантов) [1,2], а применяемая в качестве альтернативы специальная аппаратура для измерения полей концентрации нуждается в индивидуальной градуировке по каждой системе пористый материал - диффузант, что связано со значительными затратами времени и средств. Современные приборы для измерения коэффициента диффузии, основанные на контроле пространственных распределений диффундирующих веществ в исследуемых образцах, имеют высокую стоимость [3-7]. Они также нуждаются в подготовке специальных образцов для исследований, характеризуются значительными массогабаритными параметрами аппаратуры, которая нуждается в индивидуальной градуировке. Реализуемые в них измерительные процедуры характеризуются значительной продолжительностью вследствие специфики организуемых процессов массопереноса. Перспективными в плане повышения производительности измерений являются импульсные методы, получившие широкое распространение в приборах для измерения теплофизических характеристик: теплопроводности, температуропроводности и теплоемкости твердых материалов [8-10]. Однако использование их идеологии при определении характеристик массопереноса затрудняется существенной сложностью измерений локальных концентраций распределенных в твердой фазе веществ по сравнению с измерением локальных температур $[11,12]$. В связи с этим в применяемых импульсных методах измерения коэффициента диффузии для измерения локальных концентраций диффузантов используется разрезание исследуемых образцов на составляющие части $[1,13]$.

Известны технические решения, позволяющие проводить неразрушающий контроль коэффициента диффузии влаги в тонколистовых пористых материалах $[14,15]$. Однако применяемые методы предусматривают предварительную градуировку применяемых преобразователей локальной концентрации диффузанта по каждому исследуемому пористому материалу [14,15]. Это не решает проблему существенного повышения производительности контроля, так как изменение пористой структуры одного и того же материала в ходе технологического процесса его производства или эксплуатации ведет к изменению статической характеристики преобразователя. Кроме того, данные методы отличаются низкой точностью, что связано с допущением о линейности статических характеристик применяемых преобразователей локальной концентрации влаги, которые в действительности являются существенно нелинейными $[11,12]$.

В настоящей работе представлены результаты исследований коэффициента диффузии без предварительной градуировки применяемого преобразователя концентрации диффузанта в условиях неразрушающего контроля тонколистовых пористых изделий, что позволяет существенно повысить оперативность исследований. 


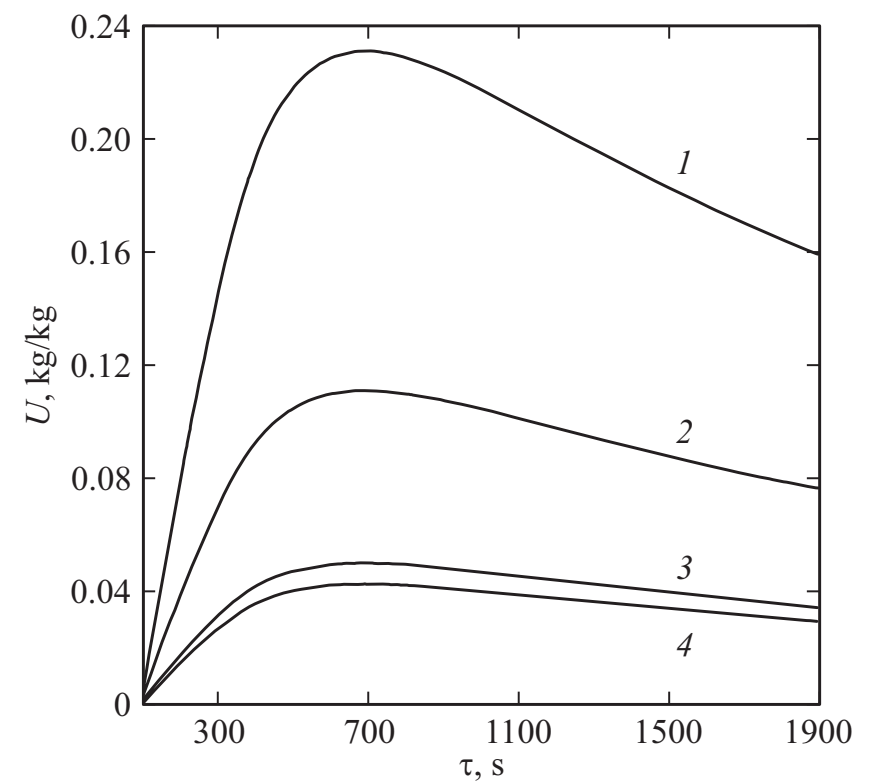

Рис. 1. Изменение концентрации этанола в фильтровальной бумаге при различных значениях дозы импульса растворителя $(\mathrm{kg}): 1-2.8 \cdot 10^{-6}, 2-2.25 \cdot 10^{-6}, 3-0.6 \cdot 10^{-6}$, $4-0.5 \cdot 10^{-6}$.

\section{Методика измерений}

Исследуемое изделие из листового пористого материала толщиной $h$ с равномерным начальным распределением растворителя помещали на плоскую подложку из непроницаемого для растворителя материала (фторопласта). К поверхности изделия плоской поверхностью в форме круга прижимался зонд, имеющий по центру отверстие для временного размещения с импульсного точечного источника дозы растворителя. На концентрической окружности радиуса $r_{0} \geq 10 h$ относительно отверстия для нанесения импульсного воздействия располагались электроды гальванического преобразователя в виде подпружиненных отрезков стальной проволоки диаметром $0.2 \mathrm{~mm}$ с нанесенным гальваническим способом покрытием цинком и медью $[11,12]$. После подачи импульса источник растворителя удалялся из зонда, отверстие для размещения источника растворителя герметизировалось заглушкой, а сам зонд обеспечивал гидроизоляцию поверхности образца в зоне действия источника и прилегающей к ней области контроля распространения растворителя. При проведении исследований длительность нанесения импульсного воздействия была более чем на порядок меньше времени достижения используемого в методе интервала реакции на него объекта. Диаметр зонда выполняли размером не менее $20 r_{0}$. Размеры плоскости подлежащего контролю изделия были достаточными для размещения измерительного зонда.

При описанной постановке эксперимента и определяющих размерах контролируемого изделия и измерительного устройства массоперенос в тонколистовом материале аналогичен распространению диффузанта в неограниченной среде при нанесении импульсного воздействия от линейного источника массы. При этом изменение концентрации растворителя на расстоянии $r_{0}$ от источника описывается уравнением [16]:

$$
U\left(r_{0}, \tau\right)=Q /\left[4 \pi D \rho_{0} \tau \exp \left(r_{0}^{2} / 4 D \tau\right)\right] .
$$

При толщине листового материала $h<10 r_{0}$ коэффициент диффузии растворителя связан соотношением [16]:

$$
D=r_{0}^{2} /\left(4 \tau_{\max }\right),
$$

где $\tau_{\max }-$ момент времени, соответствующий максимуму на кривой $U\left(r_{0}, \tau\right)$ изменения концентрации растворителя на расстоянии $r_{0}$ от точечного источника.

На рис. 1 представлены кривые изменения концентрации этанола в фильтровальной бумаге толщиной $0.2 \mathrm{~mm}$ плотностью $400 \mathrm{~kg} / \mathrm{m}^{3}$ для $r_{0}=4 \mathrm{~mm}$ при различных значениях дозы импульса растворителя. С увеличением вносимой дозы растворителя увеличивается достигаемое в $r_{0}$ значение максимума концентрации от кривой 4 к кривой 1 . Кривые имеют восходящую ветвь от начала импульсного воздействия до момента $\tau_{\max }$ и нисходящую ветвь, наблюдаемую после наступления момента $\tau_{\max }$. При этом одинаковые значения концентрации $U^{*}$, достигаемые в моменты времени $\tau_{1}$ и $\tau_{2}$ соответственно на восходящей и нисходящей ветвях кривой изменения концентрации во времени, могут быть определены из выражения (1) с учетом (2)

$$
\begin{aligned}
& U^{*}\left(r_{0}, \tau_{1}\right)=Q /\left[4 \pi D \rho_{0} \tau_{1} \exp \left(-\tau_{\max } / \tau_{1}\right)\right], \\
& U^{*}\left(r_{0}, \tau_{2}\right)=Q /\left[4 \pi D \rho_{0} \tau_{2} \exp \left(-\tau_{\max } / \tau_{2}\right)\right] .
\end{aligned}
$$

Деление (3) на (4) приводит к следующему выражению:

$$
\exp \left(\tau_{\max } / \tau_{2}-\tau_{\max } / \tau_{1}\right)=\tau_{1} / \tau_{2} .
$$

Из (5) получено

$$
\left(\tau_{2}-\tau_{1}\right) \tau_{\max }=\tau_{2} \tau_{1} \ln \left(\tau_{2} / \tau_{1}\right) .
$$

Из (6) с учетом (2) получено расчетное выражение для определения искомого коэффициента диффузии

$$
D=r_{0}^{2}\left(\tau_{2}-\tau_{1}\right) /\left[4 \tau_{2} \tau_{1} \ln \left(\tau_{2} / \tau_{1}\right)\right] .
$$

Полученное выражение использовали для определения коэффициента диффузии в отсутствие реальной статической характеристики применяемого преобразователя. Статическая характеристика гальванического преобразователя (ГП) имеет монотонный характер, поэтому одинаковые значения концентрации диффузанта и соответствующие им ЭДС преобразователя достигаются в одни и те же моменты времени. Статическая характеристика ГП характеризуется существенной нелинейностью, низкой чувствительностью к изменению концентрации при переходе растворителя из зоны связанного состояния с твердой фазой к свободному состоянию, 
Коэффициент диффузии этанола в фильтровальной бумаге

\begin{tabular}{|c|c|c|c|c|c|c|c|c|c|c|}
\hline № опыта & 1 & 2 & 3 & 4 & 5 & 6 & 7 & 8 & 9 & 10 \\
\hline$\tau_{1}, \mathrm{~s}$ & 383.2 & 448.3 & 481.2 & 493.3 & 366.8 & 501.0 & 379.3 & 392.0 & 477.6 & 400.6 \\
\hline$\tau_{2}, \mathrm{~s}$ & 1012.0 & 1183.9 & 1270.7 & 1302.5 & 968.5 & 1322.9 & 1001.5 & 1035.2 & 1261.2 & 1057.9 \\
\hline $\begin{array}{c}D_{i} \cdot 10^{9} \\
\mathrm{~m}^{2} / \mathrm{s}\end{array}$ & 6.68 & 5.71 & 5.32 & 5.19 & 6.98 & 5.11 & 6.75 & 6.53 & 5.36 & 6.39 \\
\hline $\begin{array}{c}\Delta D_{i} \cdot 10^{9}, \\
\mathrm{~m}^{2} / \mathrm{s}\end{array}$ & 0.58 & -0.39 & -0.78 & -0.91 & 0.88 & -0.99 & 0.65 & 0.43 & -0.74 & 0.29 \\
\hline
\end{tabular}

Продолжение таблицы

\begin{tabular}{c|r|r|r|r|r|r|r|r|r|r}
\hline № опыта & 11 & \multicolumn{1}{c|}{12} & \multicolumn{1}{c|}{13} & \multicolumn{1}{c|}{14} & \multicolumn{1}{c|}{15} & \multicolumn{1}{c|}{16} & 17 & 18 & 19 & 20 \\
\hline$\tau_{1}, \mathrm{~s}$ & 368.3 & 491.4 & 410.3 & 375.4 & 361.6 & 458.0 & 497.1 & 479.4 & 385.5 & 367.8 \\
$\tau_{2}, \mathrm{~s}$ & 972.7 & 1297.5 & 1083.3 & 991.2 & 954.8 & 1209.3 & 1312.6 & 1265.9 & 1018.1 & 971.3 \\
$\begin{array}{c}D_{i} \cdot 10^{9}, \\
\mathrm{~m}^{2} / \mathrm{s}\end{array}$ & 6.95 & 5.21 & 6.24 & 6.82 & 7.08 & 5.59 & 5.15 & 5.34 & 6.64 & 6.96 \\
$\begin{array}{c}\Delta D_{i} \cdot 10^{9} \\
\mathrm{~m}^{2} / \mathrm{s}\end{array}$ & 0.85 & -0.89 & 0.14 & 0.72 & 0.98 & -0.51 & -0.95 & -0.76 & 0.54 & 0.86
\end{tabular}

Примечание: в таблице использованы следующие обозначения: $\bar{D}, D_{i}, \Delta D_{i}=\left(D_{i}-\bar{D}\right)-$ соответственно математическое ожидание случайной величины и значения коэффициента диффузии и абсолютной погрешности его измерения в $i$-ом опыте $(i=1,2, \ldots, 20)$.

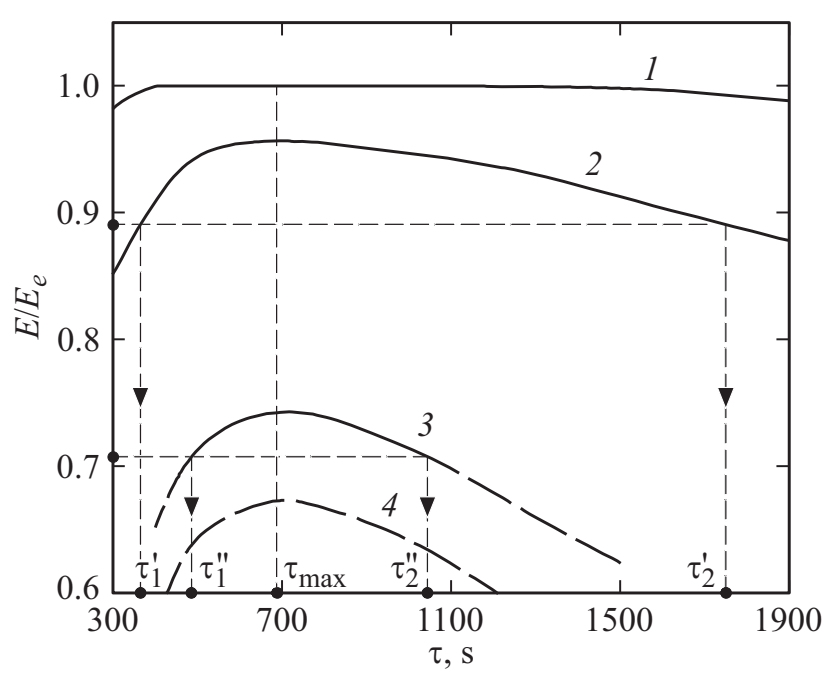

Рис. 2. Изменение ЭДС преобразователя при тех же значениях дозы импульса растворителя.

снижением помехозащищенности в области низких концентраций растворителя, соответствующим сильно связанному с твердой фазой состоянию растворителя и высокому внутреннему сопротивлению материала [11]. Но применяемая методика проведения эксперимента позволяет выбрать два произвольных одинаковых значения ЭДС преобразователя на восходящей и нисходящих ветвях кривой изменения ЭДС, соответствующих среднему участку статической характеристики ГП со стабильным помехозащищенным сигналом измеряемой ЭДС [11].

На рис. 2 представлены кривые изменения ЭДС преобразователя, соответствующие кривым изменения концентрации на рис. 1. Здесь выходная характеристика преобразователя представлена в относительных единицах по отношению к $E_{e}$, где $E_{e}-$ сигнал преобразователя, соответствующий переходу растворителя из области связанного с твердой фазой исследуемого материала в область свободного состояния (максимально возможная ЭДС преобразователя при температуре контроля). Из рис. 2 видно, что значительная часть кривой 1 изменения ЭДС в окрестности максимума концентрации (рис. 1) находится в области плато насыщения статической характеристики преобразователя с низкой чувствительностью к изменению концентрации или вообще за ее пределами, где чувствительность преобразователя вообще отсутствует. Кривая 4 на рис. 2 находится в области нестабильной работы преобразователя, что приводит к значительным погрешностям определения коэффициента диффузии. Кривые 2,3 на рис. 2 могут быть использованы для нахождения значений моментов времени $\tau_{1}$ и $\tau_{2}$ с целью расчета искомого коэффициента диффузии, согласно (7), при выбранных равных значениях ЭДС преобразователя на восходящей и нисходящей ветвях кривой изменения: $\tau_{1}^{\prime}$ и $\tau_{2}^{\prime}$ (кривая 2) или $\tau_{1}^{\prime \prime}$ и $\tau_{2}^{\prime \prime}$ (кривая 3). Таким образом, исследователь имеет возможность выбирать произвольные равные значения ЭДС преобразователя из всего рационального диапазона статической характеристики ГП $[11,12]$ в местах с высокой чувствительностью измеряемой величины к изменению времени. Это позволяет существенно снизить погрешность определения моментов времени, входящих в расчетное выражение (7), по сравнению с погрешностью определения момента достижения максимального значения ЭДС, входящего в расчетную формулу, при- 
меняемую в [16]. Опыт использования гальванического преобразователя для контроля локальных значений концентрации распределенных в твердой фазе растворителей показывает, что наилучшая чувствительность выходной характеристики ГП к изменению концентрации и стабильность его работы наблюдается в диапазоне изменения относительного значения ЭДС $E / E_{e}=0.7-0.9$. Поэтому выбор равных значений ЭДС для определения входящих в расчетную формулу значений моментов времени $\tau_{1}$ и $\tau_{2}$ целесообразно проводить из этого диапазона.

\section{Результаты экспериментов}

В таблице в качестве примера представлены результаты 20-кратных измерений коэффициента диффузии этанола в фильтровальной бумаге толщиной $0.2 \mathrm{~mm}$, плотностью в сухом состоянии $400 \mathrm{~kg} / \mathrm{m}^{3}$. Расстояние от источника дозы растворителя до расположения электродов гальванического преобразователя - $4 \mathrm{~mm}$. Доза импульса этанола составляла $\sim 9 \cdot 10^{-7} \mathrm{~kg}$. Моменты времени $\tau_{1}$ и $\tau_{2}$ определяли при относительном значении ЭДС $\sim 0.8 E_{e}$.

В настоящее время нет возможности оценить систематическую составляющую погрешности в связи с отсутствием эталонных материалов для рассматриваемого класса систем пористый материал - растворитель, поэтому проанализирована случайная составляющая. Погрешность результата измерений определяли классическим методом как половину доверительного интервала [16]:

$$
\delta D_{\Sigma}=t_{\alpha, n} S_{n} /(\bar{D} \sqrt{n})
$$

где $S_{n}=\sqrt{\sum_{i=1}^{n}\left(D_{i}-\bar{D}\right)^{2} /(n-1)}-$ средняя квадратическая погрешность отдельного измерения; $t_{\alpha, n}-$ коэффициент Стьюдента при доверительной вероятности $\alpha=0.95$ и числе опытов $n$.

В результате получены следующие значения коэффициента диффузии и погрешности его измерения: $\bar{D} \cdot=6.1 \cdot 10^{-9} \mathrm{~m}^{2} / \mathrm{s} ; \delta D_{\Sigma} \approx 6 \%$. Длительность эксперимента не превышала $23 \mathrm{~min}$.

\section{Обсуждение результатов}

Полученные результаты свидетельствуют о том, что применяемая методика позволяет сравнительно быстро измерить коэффициент диффузии в тонколистовых изделиях из пористых материалов без проведения предварительной градуировки применяемого преобразователя по каждой новой системе пористый материал - растворитель. При этом исключается необходимость разрушения изделий для изготовления специальных образцов для исследований. Погрешность результата определения коэффициента диффузии предлагаемым методом при прочих равных условиях ниже по сравнению с результатами, получаемыми при использовании метода, основанного на фиксировании момента достижения максимума концентрации при импульсном воздействии [16]. Предлагаемый метод определения коэффициента диффузии обладает патентной чистотой, на него подана заявка на предполагаемое изобретение.

\section{Заключение}

Предлагаемый метод неразрушающего контроля по сравнению с известными методами обладает гибкостью в выборе измеряемых параметров из диапазона с максимальной чувствительностью и помехозащищенностью применяемого гальванического преобразователя, что позволяет обеспечить более высокую точность определения коэффициента диффузии при соблюдении возможности не проводить длительную и затратную процедуру предварительной градуировки применяемого измерителя концентрации диффузанта.

\section{Конфликт интересов}

Авторы заявляют, что у них нет конфликта интересов.

\section{Список литературы}

[1] García-Gutiérrez M.J.L.C., Cormenzana J.L., Missana T., Mingarro M., Molinero J. // J. Iberian Geology. 2006. Vol. 32. N 1. P. 37-53.

[2] Shackelford C.D., Daniel D.E. // J. Geotechn. Engineer. 1991. P. 467-484.

[3] Janz M. Methods of measuring the moisture diffusivity at high moisture levels. Lund: Division of Building Materials, 1997. $73 \mathrm{p}$.

[4] Pel L., Kopinga K., Brocken H. // Heron-journal. 1996. Vol. 41. N 2. P. 95-105.

[5] Nizovtsev M.I., Stankus S.V., Sterlyagov A.N., Terekhov V.I., Khairulin R.A. // Int. J. Heat Mass Transfer. 2008. Vol. 51. N 17. P. 4161-4167. https://doi.org/10.1016/j.ijheatmasstransfer.2008.01.013

[6] Roels S., Carmeliet J. // Int. J. Heat Mass Transfer. 2006. Vol. 49. N 25. P. 4762-4772.

https://doi.org/10.1016/j.jheatmasstransfer.2006.06.035.

[7] Коптюг И.В., Сагдеев Р.3. // Усп. хим. 2002. Т. 71. № 10. C. 899-949. [Koptyug I.V., Sagdeev R.Z. // Russian Chem. Rev. 2002. Vol. 71. N 10. P. 789-835. DOI: 10.1070/RC2002v071n10ABEH000743

[8] Gromov Y.Y., Churikov A.A., Divin A.G., Ishchuk I.N., Barkalov S.A. // J. Engineer. Sci. Technol. Rev. 2016. Vol. 9. N 6. P. 135-141.

[9] Селиванова 3.М., Хоан Т.А. // Изм. техника. 2015. № 9. C. 45-48. [Selivanova Z.M., Khoan T.A. // Measurement Techniq. 2015. Vol. 58. N 9. P. 1010-1015. https://doi.org/10.1007/s11018-017-1220-5]

[10] Гуров А.В., Соседов Г.А., Родина А.Е., Пономарев С.В. // Изм. техника. 2012. № 10. С. 47-49. [Gurov A.V., Sosedov G.A., Rodina A.E., Ponomarev S.V. // Measurement Techniq. 2013. Vol. 55. N 10. P. 1187-1192.] 
[11] Мищенко С.В., Беляев П.С., Фролов А.П. // Метрология. 1988. № 8. C. 55-61.

[12] Mishchenko S.V., Belyaev P.S., Gladkikh V.A., Safronova E.N. // Drying Technol.1999. Vol. 17. N 10. P. 2151-2167.

[13] Мищенко С.В., Пономарев С.В., Толстых С.Г., Толcmыx C.C. // Вест. Тамбовского гос. техн. ун-та. 2003. Т. 9. № 2. C. $150-165$.

[14] Мочалин С.Н., Исаева И.Н., Пономарев С.В. // Вест. Тамбовского гос. техн. ун-та. 2010. Т. 16. № 3. С. 533-545.

[15] Мочалин С.Н. // Вопросы современной науки и практики. Университет им. В.И. Вернадского. 2010. № 7-9 (30). C. $329-337$.

[16] Беляев В.П., Беляев М.П., Мищенко С.В., Беляев П.С. // Изм. техника. 2013. № 10. С. 65-69. [Belyaev V.P., Belyaev M.P., Mishchenko S.V., Belyaev P.S. // Measurement Techniq. 2014. Vol. 56. N 10. P. 1190-1196.

DOI: $10.1007 / \mathrm{s} 11018-014-0353-\mathrm{z}]$ 